\title{
A Review of Some Assistive Tools and their Limitations for Visually Impaired
}

\author{
${ }^{*}$ Suresh Merugu, ${ }^{2}$ George Ghinea, ${ }^{3}$ Abdul Subhani Shaik, ${ }^{4}$ Manir Ahmed, ${ }^{5} \mathrm{P}$. Raveendra Babu, \\ ${ }^{5}$ M. Nagaraju Naik \\ 1,3,4,5 Department of ECE, CMR College of Engineering \& Technology, \\ Hyderabad, Telangana, India \\ ${ }^{2}$ Mulsemedia Computing, Wilfred Brown Building 215, Brunel University, London, UK \\ msuresh@cmrcet.org
}

Received: $8^{\text {th }}$ November 2021, Accepted: $5^{\text {th }}$ January 2022, Published: $28^{\text {th }}$ February 2022

\begin{abstract}
World report on Vision by World Health Organization reported that there are at least 2.2 Billion people are Visually Impaired. "The National Blindness \& Visual Impairment Survey India 2015-2019" survey report by Government of India reported that the overall estimated prevalence of the Blindness in age 50 or more is $1.99 \%$ and the overall estimated prevalence of Visually Impaired in age 50 or more is $13.76 \%$. The above numbers urge researchers to develop advanced assistive tools for Blind and Visually Impaired. There have been several assistive tools already designed to assist and enhance the quality of life to Blind and Visually Impaired. But most of them are limited and confined to specific problems. In this paper, a systematic review of some of the assistive tools for Blind and Visually Impaired is done. The detailed review of existing assistive tools reveals that the factors like user-friendly, ensuring safety, independent mobility, multiple features and user-centric design need priority consideration while designing such tools.
\end{abstract}

Keywords: Assistive tools, Assistive technology, Blind, Visually Impaired, Independent Mobility, Travel aids, Wearable devices, Safety, User-centric design

\section{Introduction}

A person with vision disability in most of the cases has to rely on other senses and depend on others to perform their daily routine tasks. While performing these tasks, Visually Impaired persons come across various challenges that affect the quality of their lives. World report on Vision by World Health Organization reported that there are at least 2.2 Billion people are Blind or Visually Impaired [11]. The Ministry of Health and Family Welfare, Government of India conducted a survey for more than 85,000 people "The National Blindness \& Visual Impairment Survey India $2015-2019$ " recently. The overall estimated prevalence of the Blindness in age 50 or more is $1.99 \%$ and the overall estimated prevalence of Visually Impaired in age 50 or more is $13.76 \%$ was observed in the survey [12]. The above facts show that there is a significant number of individuals facing vision problems.

In order to assist, enhance and improve the quality of all such individuals, there is a great need of development of advanced assistive tools for Blind and Visually Impaired persons. Assistive tools with multiple features will have great impact on their lives and will enhance the capabilities of Blind and Visually Impaired. But the key is assistive tools developed should be user-friendly, address all the problems of the users and ensure the primary requirements like safety and independent mobility of the users.

The following review of Literature presents the outcomes and the limitations of some of the existing assistive tools 
for Blind and Visually Impaired and the various factors that need to be considered while designing the devices.

\section{Assistive Technology}

Assistive tools are developed to enhance the capabilities of the disabled persons. Assistive technology is broadly categorized as Low-technology and High-Technology. Some of the popularly known low-tech aids are crutches, reading glasses and hearing aids. With the great progress in Science and Technology in the recent past, others are more advanced by integrating the smart phone and computer access [8].

In the past, the assistive technologies mainly concentrated on two problems Information Transmission and Mobile Assistance of the Blind and Visually Impaired. Problems related to Information Transmission are identifying characters, reading and recognizing graphical representations. These problems are addressed in most of the cases by Braille dot code and Tactile displays. Problems related to Mobile Assistance are involved in environment perception, orientation and obstacle avoidance. These problems are addressed by scanning the environment using various technologies and communicating the collected information to other senses through audio or tactile displays. In this modern internet world, problems to access computers arose. These problems are addressed by Voice synthesizers, Screen Magnifiers and Braille output terminals.

Addressing the problem along with ease to use in a user-friendly and comfortable way will enable the user to use the device to the fullest. There are two major types of assistive tools namely, portable and Wearable.

Portable devices are compact, lightweight and easy to carry and require constant hand interaction like laptops, electronic canes, and tactile displays. Wearable devices allow hands-free or minimal interaction while using the device. These are usually worn on head, wrist, waist etc. In view of Blind and Visually Impaired persons, Wearable devices give great comfort [7].

\section{Portable Electronic Travel Tools}

Mounir Bousbia-Salah, Mohamed Fezari [2] proposed a portable Electronic Travel Aid device that provides independent mobility and let travel through known and unknown environments to blind and visually impaired pedestrians without others assistance. It provides urban walking routes information using spoken words to indicate what decisions to make.

The aid consists of microcontroller, an accelerometer, a footswitch, a speech synthesizer, a push-button switch, a mode switch and a power switch. The system has two modes of operations namely Record and Playback. Record mode: In this mode, the system records the distance travelled by the user in the route. When the user reaches a decision point, for example if the route takes a left turn then the user gives an input by pressing a key on the aid. This will lead to two effects in system. First, distance travelled by user is stored in memory of the Microcontroller and counter is reset to zero. Second, left turn instruction is recorded as a decision word within the speech synthesizer. Playback mode: When the distance travelled by the user is equal to that stored in memory for that particular route then corresponding decision word is generated by synthesizer is given as speech output to the user.

Electronic Travel Aid will enhance the independent mobility of visually impaired persons. Acceleration of the user is used to measure the distance travelled by using an Accelerometer and two integrators. Using footswitch overcomes drift problems in this system. It is desirable to use this aid with the ISD5216[13] speech synthesizer, which is a record/playback device that would give the blind a direct access to more routes. Electronic Travel Aid involves a microcontroller which can assist the blind persons about walking routes by using speech synthesizer to point out what decisions to make. It helps to explore the electronic map and allows planning optimum route to the 
desired destination. Obstacles detection system [14] detects obstacles and other hazards to help visually impaired persons to travel safe and quick.

The major drawback of the system is Global positioning system (GPS), the user position information will be investigated in the future.

Piyush Chanana, Rohan Paul, M Balakrishnan and PVM Rao [9] made a comprehensive study on various assistive technology solutions available for Visually Impaired people and the study revealed that most of the assistive technologies already present provide limited solutions to the user needs. Most of them focused on technology and not on user needs.

Designing of any assistive tool should involve the user in throughout the design and development process. Regular feedback and inputs from the beginning till the end from the user will address maximum needs. Incorporating assistive technologies in the most commonly used devices like watches, portable computers and mobile phones will give best results. Keeping in mind, about the retention capabilities of the brain, the information communicating to the user should be short, understandable and in the apt format. Even though correct information is collected from the environment, the key is to communicate the same in the exact way to the user through user friendly human-computer interface.

Obstacle detection systems make use of ultrasonic sensors, laser, cameras, infrared. The best approach that addresses most of the problems in detecting obstacles is to use multiple obstacle detection devices. Obstacle detection devices in spectacles will detect overhanging objects, hand held detection devices detect large objects and detection devices in cane will address holes, bumps on the roads, drop-offs issues. There are many navigation solutions available and each has its own procedures and constructions. The requirement of the user is key and a very few devices support both indoor and outdoor environments. Most of the GPS applications are designed for vehicles, pedestrians and provide visual feedback. These applications with focus on assisting blind will help. Cane-mountable obstacle detection systems with smart phone-based navigation solutions will provide multiple solutions to the visually impaired in an economic and customizable simple user interface. Radio Frequency Identification (RFID), Wi-Fi, Bluetooth based devices are the better options for navigation in indoor environments. Thorough study of navigation information needs and user preferences needs will help the users greatly.

\section{The Major Limitations are:}

1. In addition to the regular functionality, GPS based navigation devices should provide the information like bumps on the roads, footpaths, road crosses etc which are more relevant/helpful to Visually Impaired.

2. Inaccuracies in GPS based devices will not let the users reach their desired destinations. Inaccuracies should be minimized to negligible.

3. Navigation devices that enable users to customize the familiarised routes will help.

\section{Mobile Accessibility Tools}

Lilit Hakobyan, Jo Lumsden, Dympna O'Sullivan, Hannah Bartlett [4] did research in the mobile assistive technologies for Visually Impaired and to maximize the Mobile phones access to Visually Impaired persons. To align technology and medical expertise to meet the requirements of the end user.

Designing of assistive tools in user-centered design can be achieved successfully by including surveys, interviews, usability testing. Various mobile assistive technologies were discussed in the paper but most of the systems discussed in the paper are in under development stage.

Using mobile devices as mobile assistive technology for Visually Impaired provide various advantages like device can be accessed at any time from any place, portable, not attracting attention of others, mainstream device and users 
feel less stigmatized. Mobile assistive technologies enhance the capability of Visually Impaired to live independently and to participate in social events. Unlike conventional assistive aids, mobile assistive technologies provide Visually Impaired people an advantage of lightweight, portable, discrete aids delivered through the devices that are well known to common people, leading to feel less stigmatized. Mobile assistive technologies with usercentered design (UCD) and by engaging all stakeholders will focus on users with different needs, capabilities and attitudes which result in fulfilling maximum needs of the user.

\section{The Major Limitations presented are:}

1. Assistive technology without user inputs/opinion may not last for long and may lead to technology abandonment.

2. Mobile assistive technologies are developed to specific requirements like MoBraille, Voice Maps, Rampe, ShopMobile-2 etc

A dissertation submitted by Nektarios Paisios [5] explains the complete design, implementation and evaluation of four mobile applications that assist Visually Impaired people overcome barriers in their daily life. The four applications studied are designed for: A mobile tool for independent way-finding, typing on touch screen using Braille, reliable currency identification and pattern matching for clothes.

The Navigational guide system was tested on users of sample size nine, out of which 8 are totally blind and 1 is partially sighted. The sample comprised of different categories like young and old, novice and expert users in navigation systems. The feedback of each user about the system was recorded through a questionnaire. Mobile software for different prototype methods to enter text in mobile using touch screen based on Braille alphabet for blind test was conducted on 15 participants. A Mobile tool for reliable cash identification, the system could classify the currency perfectly when the images were properly taken.

Navigational guide device is a significant step that helps Blind and Visually Impaired to navigate in indoor environment. The Mobile software for typing on touch screen using Braille test results indicate that most of the users preferred spatially-oriented method of entering Braille using single finger. Modern Smart phones with high resolution cameras with mobile currency recognition enhanced the ability of Visually Impaired to exchange the cash correctly and effortlessly. Regarding patter matching tool for clothes, it is observed that the standard neural network performs better than Ridge regression algorithm.

The Major Limitations presented are:

1. The Navigational guide system testing result convey that the system works fine in short routes and limited complexities but doesn't work as expected sometimes due to Wi-Fi fluctuations.

2. In typing on touch screen using Braille, the noisy touch issues should be minimized.

3. Currency recognizer will work only when one currency bill is scanned.

4. More work needs to be done like finding more samples for other types of clothes, other characteristics like texture and design patterns.

WafaElmannai and Khaled Elleithy [10] presented a detailed survey to address and present the issues in the portable and assistive vision substitution devices for Visually Impaired people to pave a way to other researchers to design new devices that resolve the current issues and provide safe and independent mobility to visually impaired people.

A comprehensive study of various reviewed systems is evaluated based on the basic parameters like analysis type, coverage, time, range and object type. Each parameter is graded in the range 0 to 10 . A normalization formula is used to calculate the total score of each parameter. In the complete examination, systems that support autonomous navigation feature stood in the first place. By this survey, it is observed that many devices don't fulfil all the 
requirements of the users. Depending on the user's feedback and study, an evaluation of all systems is done and respective limitations/areas to improve each system are presented.

The study highlights the importance of essential guidelines that need to be followed to design assistive devices to ensure user satisfaction. A few aspects that need to be followed are user friendly, wearable, affordable, reliable, high performance and wireless connectivity. It is clear evident that none of the examined devices provided complete support to the evaluated features that are mentioned in the study and the reason observed is missing fundamental features.

\section{The Major Limitations presented are:}

In the survey conducted, it is known that almost 7 out of 10 devices fulfil the following three major factors partially.

1. Devices supporting both indoor and outdoor environments.

2. Obstacle detection range from user is less than 5 meters due to ultrasonic limitation.

3. Inability to detect dynamic objects.

\section{Wearable Assistive tools}

Rohit Takhar, Tushar Sharma, Udit Arora, Sohit Verma [1] developed a low-cost wearable assistive product using a webcam and Raspberry Pi that assists Visually Impaired people in detecting and recognizing pedestrians' crosswalks and staircases and the device is capable to adapt to different light conditions.

Through Raspberry Pi Wi-Fi hotspot connection is established with the Smartphone. Images of obstacles are captured by a web camera. Captured images contrast is increased by applying Histogram equalization. Segmentation of images highlight black \& white patterns and it helps in differentiating road crossings from staircases. Ellipses are drawn over the contours found in the examined frame. After finding out the distance between each ellipse they are arranged in increasing order. If the longest increasing subsequence is found greater than four then road crossing prediction is made, a staircase is predicted. Android phones receive a UDP packet whenever a road cross/staircase is identified. An audio output is generated and sent to the user. This system enables Visually Impaired to overcome object detection (crosswalks and stairs) while navigating with a portable product instead of taking traditional aids like White cane and Guide dog. The test results of the system are satisfactory. The main drawback of the system is more confined in detecting crosswalks and stairs.

Ramiro Velázquez [7] worked on a study of the latest approaches and to understand universal design concepts for development of wearable assistive devices for blind. Popular design concepts of assistive devices for blind are explained below:

Assistive devices worn on fingers and hands: Bandage-sized [15] tactile display, the device has 20 contact points and tactile feel is achieved by these contact points. Different patterns like vibration, upward, downward are generated using external user computer interface. Japanese Finger-Braille interface, in this system three fingers in each hand are considered as Braille dots. Three motors on each hand are attached and these are controlled by a Citizen-IBM wrist watch computer and electronic module which has batteries and control circuitry. Wrist watches computers can establish connection with external devices through bluetooth.

Assistive devices worn on the wrist and forearm: Assistive devices worn on forearm and wrist [16]. They are simple devices that give an alert to the user when approaching an obstacle. Braille watch is a wearable assistive device that is worn on the wrist. These watches work almost similar to the regular watches but the only difference is the time is displayed in the Braille numbers.

Assistive devices worn on the tongue: Tongue Display Unit(TDU) [17] converts the images captured by camera into electro tactile stimuli and are sent to dorsum of the tongue through a flexible electrode array which is placed on the tongue. 
Head-mounted assistive devices: Sonic Guide/Kaspa System or most of the similar devices [18-19] use laser or ultrasonic beam is emitted to identify an obstacle and its distance. Once the beam is reflected back to the user then the same is communicated to the user with an audio or tactile output. Voice system uses a camera for input information. It then transforms the image information to sounds. Image position, brightness etc will differentiate frequency and loudness. Intelligent Glasses has two stereo cameras fixed to the frame of the glasses. These cameras capture the surrounding environment then obstacles are identified by applying vision algorithms. The result is communicated to the user on a tactile display.

Assistive devices worn on Vests and Belts: There are various vests devices designed. These are commonly designed with vibrating elements/motors, sensors and tactile displays. Once the sensors detect the object, they give respective instructions (like forward, back, left, right, slow, speed) to the user.

Assistive devices worn on the feet: Vibrators are integrated in regular shoe foam. These vibrators are controlled by specific vibrating frequency commands and enable them to produce vibration frequencies specific to turn left, turn right, go forward, go backward, stop and the same are communicated to the user. Since blind doesn't have visual sense, hearing and touch become the important senses. Designers should ensure that the devices don't affect these senses. Hearing a sound by humans depends on two parameters: sound intensity and frequency. Designers designing these assistive devices should consider these parameters and ensure that these parameters are within the apt range that provides hearing as an alternative sense to the visually impaired. Designing of wearable touch stimulation devices like tactile displays should be designed on the basis of two-point discrimination threshold (TPDT). TPDT is a measure that describes the range of the pressure points that are realized as two distinct points on the skin.

\section{Integration Of Different Assistive Technologies}

SándorTihamér BRASSAI, László BAKÓ, Lajos Losonczi [3] presenteda device to enhance mobility and provide technology assistance to Visually Impaired people. Various Head Related Functions are used to give inputs to the device through Computer interfaces. The proposed system has two modules and a real-time wireless communication is established between two modules.

Personal computer checks the movement of the user and records if the person has reached destination/blocked in his movement. This module supports interface for navigation, information, entertainment. The interfaces of a personal computer store the database of environment, trajectory, the current position of the user is verified against the stored information in navigation environments interface and provides access to the information technologies. Some of the major information technologies which can be accessed are Books, Computers and Documents. Allow users to access entertainment applications like play music through interface.

Portable equipment performs various functions and uses embedded systems of control and processing. Based on the object distance, sound frequencies and head orientation, respective Head Functions are implemented in this module. Artificial neural networks are used to implement HRTF functions. Input interface can contain Braille keyboard or voice input. User gives input commands based on the requirement and that is addressed by the computer. Ex: Play Music (entertainment interface in personal computer). By combining measurement data of various types of sensors by Sensor fusion module, better results can be achieved to track the user location. Based on the detection and non-detection of obstacles; respective instructions are communicated to users through the ManMachine interface. Obstacles in the environment are indicated by beep sound and are differentiated by the frequency of sound. To identify objects and measure distance, a system that takes input from multiple sensors is an important aspect. Artificial Intelligence and Neural Network plays key roles in adapting to familiar and unfamiliar environments at run time. 
Assistive technologies for Visually Impaired are classified into two categories - Daily life (personal care, general health, food) and Navigation \& Orientation.

Technologies for Daily life: Tactile Labeling Systems (using alphabets that are used by blind like Braille, Moon and Fishburne alphabets), Radio Frequency Labeling Systems, Radio Frequency Identification (RFID) which retrieves remote data automatically using RFID tags, Barcode Labeling technologies usage with voice synthesizer are suggested for personal care needs. Regular health care needs like BP checkup, body temperature, weight and so on are assisted by headset audio and tactile display output devices. Food preparation and consumption problems are addressed with the new user-centric design of kitchen items (with audio output) that ensure the safety and comfort to visually impaired. Electronic money identifying devices that accept multiple or at least most commonly used currency will give independence to manage money.

Technologies for Navigation \& Orientation: Electronic Travel Aids are built with multiple smaller components like obstacle detection system, path planner module, user-interface and a monitoring system module. Path planner module generates the route to the desired destination which has no obstacles.3D obstacle system is responsible for detecting the positions of obstacles. Man-computer interface extracts environment information and directs Visually Impaired people accordingly. Localization and object detection are done by the sensorial interface by using infrared sensors, magnetic sensors, inertial measurement unit, sonar systems and stereo vision system. Monitoring system module track and help the user to reach the target and to help the user if there is any change in the environment. Artificial neural networks are proposed for modeling the environment, distance to the object is known in real time in the neural networks. Depending on the environment modeling outcomes, an appropriate decision is made and the same will be communicated to the person using through user interface.

\section{The Major Limitations presented are:}

1. Most of the systems using GPS sensors are not fulfilling the complete requirements of Visually Impaired people and are limited to external environments.

2. Issues related to urban areas should be resolved.

\section{Current And Future Assistive Technologies}

Philip Boucher [8] did a study on the current and future assistive technologies for Blind. Current Assistive Technologies for Blind and Visually Impaired is categorized as primary and secondary aids. Primary aids are the low-tech conventional devices like Braille texts, white canes etc which are used for reading text and navigating safely and independently. Secondary aids in most cases are used with primary aids. Different assistive technologies perform different tasks to blind and Visually Impaired based on the requirement like magnifying, transforming information into other sensory modalities like touch, reading and sound. Bionic eyes, a device which is under development is designed to convert light to electrical impulses. Augmented reality spectacles help Blind and Visually Impaired by filtering colours, magnifying objects and provide features like object recognition assistance by audio.

Future Assistive Technologies for Blind and Visually Impaired tools with minimized size, portable, better wear ability, efficient, user friendly interface enable users to use the advance touch screens will give an advantage to the Blind and Visually Impaired.

The study revealed that assistive technologies are to play a key role in assisting the lives of the disabilities and an increase in usage of assistive technologies to help disabled. An assistive technology with simple design that fulfils the requirements of a variety of users is required. Gap between assistive and mainstream technologies should be minimised. 


\section{The Major Limitations presented are:}

1. Involvement of disabilities in design and development of assistive technologies should be from the beginning.

2. Individuals have multiple disabilities with multiple requirements, personal needs and preferences. Users cannot be discriminated against with a common design assistive tool for all.

Dana A. Draa [6] presented current and future trends in technologies for the Blind and Visually Impaired. Assistive technologies enhance the capability of the Blind and Visually Impaired. These assistive technologies let Visually Impaired accomplish certain tasks which couldn't be done individually.

Current technologies used are Video magnifiers, Computers systems with external hardware and softwares, Sonic cane, Navigation bracelet, Haptic shoes, Victor reader stream, Mini-Guide, portable devices like iOS devices with certain apps can be used as video magnifiers (connecting to External Monitor/TV), Apps for iOS. New technologies, tele-health is a health service provided to the Blind and Visually Impaired person who has transportation problems.

\section{The Major Limitations presented are:}

1. Limited applications and more job-oriented applications should be introduced.

2. Less training resources, a very few people are trained.

\section{Conclusion}

The designing part of the assistive tools is the key and the purpose of these assistive tools is to address the problems of the user. This can be achieved by following User-centric approach, which gives high priority to the user needs in designing the assistive tools and give quality service to the users.

Most of the existing assistive tools are specific to certain features. Unfortunately, assistive tools addressing specific problem or with limited features are replaced with other devices in short span of time. Assistive tools with multiple features can be used to the maximum. Effective use of existing advanced technologies like features in Smart phones and integrating these features with assistive tools would give better results.

It is a positive sign to see a great progress in the assistive tools developed in the recent years. However, emphasis on design, multiple features, using of advanced technologies and ensuring the primary requirements like user-friendly, ensuring safety and independent mobility will have a great impact and reach maximum Blind and Visually Impaired persons. This paper provides a brief review on some of the Assistive Tools and their Limitations for Blind and Visually Impaired Persons but further research is required to identify and reduce the problems in assistive tools. 


\section{References}

[1] Rohit Takhar, Tushar Sharma, Udit Arora, Sohit Verma, "Assistive guidance system for the visually impaired" 2017.

[2] Balakrishna, S., Solanki, V. K., Gunjan, V. K., \& Thirumaran, M. (2019, January). Performance analysis of linked stream big data processing mechanisms for unifying IoT smart data. In International Conference on Intelligent Computing and Communication Technologies (pp. 680-688). Springer, Singapore.

[3] SándorTihamér BRASSAI, László BAKÓ, Lajos LOSONCZI, "Assistive Technologies for Visually Impaired People" Electrical and Mechanical Engineering, 3 (2011) 39-50

[4] Lilit Hakobyan, Jo Lumsden, Dympna O’Sullivan, Hannah Bartlett, "Mobile Assistive Technologies for the Visually Impaired".

[5] Balakrishna, S., Solanki, V. K., Gunjan, V. K., \& Thirumaran, M. (2019, January). A survey on semantic approaches for IoT data integration in smart cities. In International Conference on Intelligent Computing and Communication Technologies (pp. 827-835). Springer, Singapore.

[6] Ramiro Velázquez, "Wearable Assistive Devices for the Blind" Chapter 17 in A. LayEkuakille\&S.C.Mukhopadhyay (Eds.), Wearable and Autonomous Biomedical Devices and Systems for Smart Environment:Issues and Characterization, LNEE 75, Springer, pp 331-349, 2010.

[7] Philip Boucher, Scientific Foresight Unit (STOA), PE 603.218, Directorate-General for Parliamentary Research Services, "Assistive technologies for people with disabilities".

[8] Chauhan, R., Singh, R., Saraswat, A., Joya, A. H., \& Gunjan, V. K. (2014). Estimation of software quality using object oriented design metrics. International Journal of Innovative Research in Computer and Communication Engineering, 2(1), 2581-2586.

[9] Merugu, S., Reddy, M.C.S., Goyal, E., Piplani, L., "Text message classification using supervised machine learning algorithms", Lecture Notes in Electrical Engineering, 2019, Vol. 500-Issue, PP-141-150.

[10] World Health Organization, "World Report on Vision" https://www.who.int/publications-detail/worldreport-on-vision, Date: 04-04-2020.

[11] Singh, R., Chauhan, R., Gunjan, V. K., \& Singh, P. (2014). Implementation of elliptic curve cryptography for audio based application. International Journal of Engineering Research \& Technology (IJERT), 3(1), 2210-2214.

[12] Merugu, S., Jain, K., Mittal, A., Raman, B., "Sub-scene Target Detection and Recognition Using Deep Learning Convolution Neural Networks", Lecture Notes in Electrical Engineering, 2020, Vol. 601-Issue, PP1082-1101.

[13].G. Whitney, "Electronic Mobility Devices or Obstacle Detectors for Blind and Partially Sighted People," Joint Committee on Mobility of Blind and Partially Sighted People, JMU, RNIB, UK. 1994.

[14] Koo I, Kwangmok J, Koo J, Nam J, Lee Y, and Choi H (2008) Development of soft actuator-based wearable tactile display. In: IEEE Transactions on Robotics, vol. 24,no. 3, pp 549-558.

[15] Ng G, Barralon P, Dumont G, Schwarz S, Ansermino J (2007) Optimizing the tactile display of physiological information: vibro-tactile vs. electro-tactile stimulation, and forearm or wrist location. In: Proc. of Annual International Conference of the IEEE Engineering in Medicine and Biology Society, Lyon, France, pp 4202-4205.

[16] Bach-Rita P, Kaczmarek K, Tyler M, and Garcia-Lara J (1998) From perception with a49-point electrotactile stimulus array on the tongue: a technical note. In: Journal of Rehabilitation Research and Development, vol. 35 , no. 4, pp 427-430.

[17] Merugu, S., Tiwari, A., Sharma, S.K., "Spatial Spectral Image Classification with Edge Preserving Method", Journal of the Indian Society of Remote Sensing, 2021, Vol. 49-Issue 3, PP-703-711.

[18] Yadav, B.C., Merugu, S., Jain, K., "Error assessment of fundamental matrix parameters", Lecture Notes in Electrical Engineering, 2019, Vol. 500-Issue, PP-151-160.

[19] Velazquez R, Bazan O, and Magaña M (2009) A shoe-integrated tactile display for directional navigation. In: Proc. of IEEE/RSJ International Conference on IntelligentRobots and Systems, St. Louis, MO, USA, pp 1235-1240. 